\title{
A Bench Set-Up for Low-Beta Beam-Current Test Measurements with COSY
}

\author{
Ch. Günther, A. Schnase, and H. Meuth \\ IKP, Forschungszentrum Jülich GmbH, Postfach 1913, D-52425 Jülich \\ F. Caspers \\ PS Division, CERN, CH 1211, Geneva 23
}

\section{Abstract}

Beam current bench tests and simulations, e.g. with beam monitors, are of interest for diagnostic purposes. For highlyrelativistic tests, the common coaxial wire method may be used. In contrast, a configuration for non-relativistic beams was devised for transverse impedance measurcments. We have combined the two approaches to permit both frequency and time domain low-beta bench tests of the line charge, and of the longitudinal beam impedance. The basic structure is of coaxial $50 \Omega$ geometry, where the outer diameter matches the beam-pipe diameter of $C O S Y$, while the inner conductor carries, both around the azimuth and along the axis, suitable pinand-loop arrays for imposing electric and magnetic ficlds, respectively. The arrays ensure nearly azimuthal (coaxial) symmetry, and are wired via delay lines such that axially varying field patterns may be generated, permitting the simulation of a broad beta range. We use standard measurement techniques with a network analyzer at a frequency range from $500 \mathrm{kHz}$ up to $100 \mathrm{MHz}$. A calibration procedure, measuring field pickup via coupling loops and pins installed in the outer beam pipe wall, ensures the correct $|\mathrm{El} / \mathrm{B}|$ ratio.

\section{INTRODUCTION}

The coaxial-wire method is commonly employed for simulating a ficld configuration inside, the beam pipe, that is similar to fields gencrated by a highly relativistic beam, i.e. $\beta \sim 1$, where $\beta$ is the ratio of the beam velocity to the speed of light in vacuum. In this case fields are closely approximated by modes of TEM-type. Correspondingly, transverse highly relativistic modes are suitably modelled by the common two-wire method, driven in balanced-line, or, respectively, the singlewire-plus-ground-plane method. $[1,2]$ To approximate the situation of transverse modes of a low-beta beam more realistically, a delayed loop-and-pin structure was devised, incorporating in addition a metallic symmetry-plane. [2,3] This structure was used to characterize a meander-shaped slow-wave pick-up structure used for stochastic cooling at low beta.

Here, we present a similar set-up, although topologically different in approach, that aims at the simulation of longitudinal fields of low-beta beams and at the characterization of impedances of suructures excited by such beams. The planar geometry of reference $[2,3]$ cannot be used in a straightforward manner for this purpose. Rather, small loops and pins are placed on the inner conductor both around the azimuth and along the axis of the beam pipe. This (quasi-cylindrical) geometry is less accessible to the quantitative description, than is the planar case. $[2,3]$ For instance, an azimuthally symmetric purely azimuthal magnetic field configuration in between inner and outer conductors cannot be produced by an azimuthally symmetric current distribution on the inner conductor alone. The magnetic fields in between inner and outer tube, therefore, essentially depend on the azimuthal array of discrete loops.
As an approximate tool to estimate the respective field configurations, we therefore started from single loop and pin propertics.

\section{MECHANICAL CONFIGURATION}

Starting from a basic coaxial structure of a $50-\Omega$ geometry, as it is used for relativistic test measurements, the outer diameter matches the beam-pipe diameter of COSY $(150 \mathrm{~mm})$, while the inner conductor must thus measure $66 \mathrm{~mm}$ in diameter. The inner conductor is divided into mating modules, cach carrying either pins or loops to impose, respectively, electric or magnetic fields. The inner tube serves as the ground/reference surface for the pin-and-loop arrays.

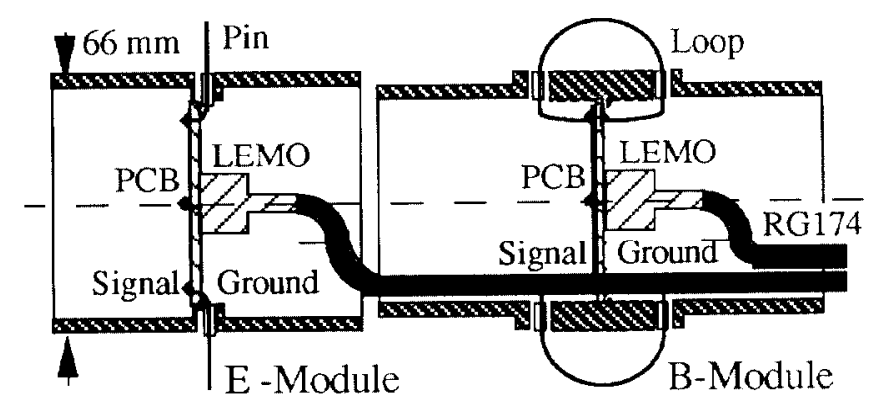

Fig. 1: Schematic of the modules for loops and pins

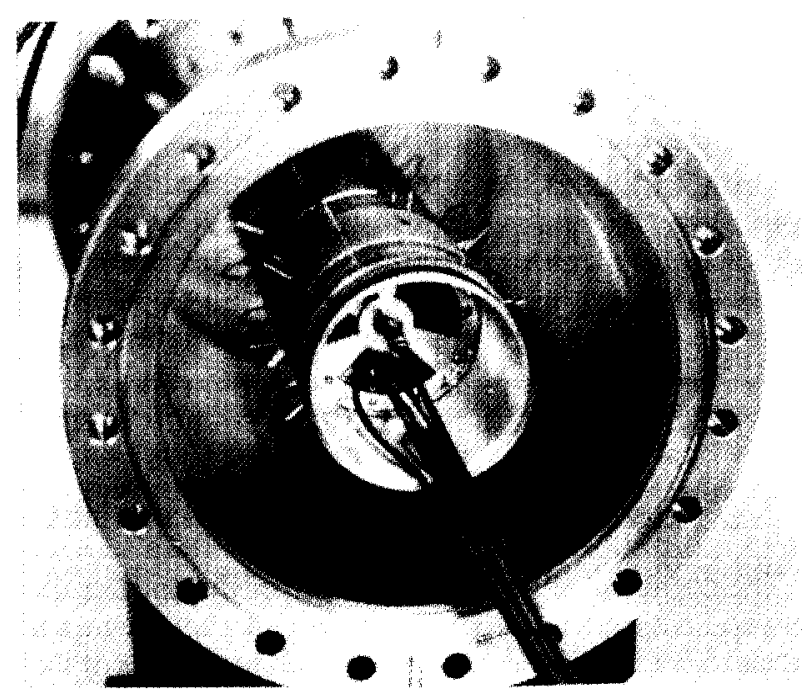

Fig. 2: Photograph of actually assembled loop and pin arrays

The pins consist of rods of $10 \mathrm{~mm}$ length, and the loops of a semi-circle curved of $10 \mathrm{~mm}$ radius. Both pins and loops are formed from semi-rigid $1-\mathrm{mm}$ diameter wire, with the tefIon insulation $(0.5 \mathrm{~mm}$ thick) stripped back, where they ex- 
tend from the inner conductor. There are 10 pins and 10 loops installed evenly azimuthally into each inner tube module. The modular construction allows for easy assembly and wiring of each station, and for configurational flexibility, achieving a minimum loop-to-pin distance of $\lambda_{\min } / 2=35 \mathrm{~mm}, \lambda$ being the structure periodicity (and the field distribution periodicity, if all loops were to be driven in phase). With our module design, this length may arbitrarily be extended by inserting matching extension tubes. Modules are mechanically plugged together; each module features a conical tight fit ensuring mechanical stability of the inner tube without the use of further mechanical fixtures. Conceivably, the minimum distance of $35 \mathrm{~mm}$ could be reduced, if a different joining mechanism were to be used. In this respect, we should note, that with these lengths, wc roughly fulfill the scale length inequality given by Equ. (9) of [3], which should, at least approximately, also apply to our case. Finally, within each module, pins or loops are connected electrically and mechanically by a suitably shaped printed circuit board (PCB), on which a LEMO connector was installed for attaching the external signal (delay) line.

\section{ELECTRICAL CHARACTERIZATION}

Electrically, pins are left "dangling" free, while the far end of the loops are connected to the ground/reference plane of the circuit board, to which, in turn, both the inner conductor tube, and the outer conductor/shield of the LEMO is attached (Fig. 1). To characterize a naked pin or loop, they were mounted on the respective PCB, separate from their inner conductor modules. We determined the capacitance and inductance in dependence of the number of loops/pins attached (1 to 10), and found, for the naked $\mathrm{L}_{\text {loop }}=40 \mathrm{nII}$, and $\mathrm{C}_{\mathrm{pin}}=2.87 \mathrm{pF}$. The loops showed a slightly nonlinear behavior, presumably due to loop-loop coupling. In their final assembly in the module, the value for the single pin capacitance and loop inductance were, at $10 \mathrm{MHz}, \mathrm{C}_{\mathrm{pin}}=3.65 \mathrm{pF}$ and $\mathrm{I}_{\text {loop }}=39 \mathrm{nH}$.

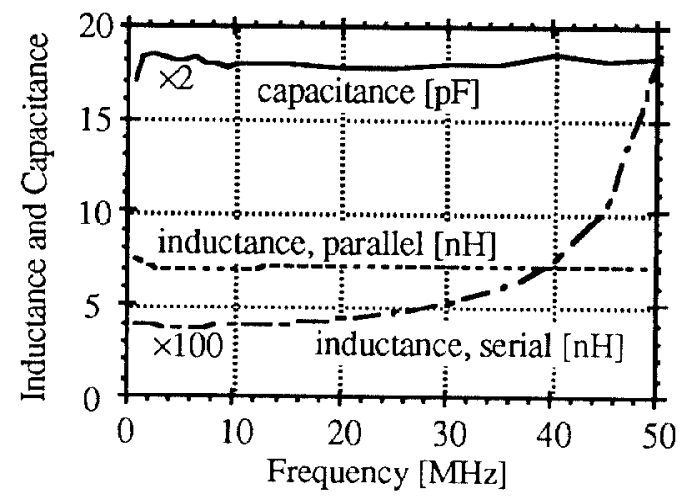

Fig.3: Total loop inductance/pin capacitance of module

The capacitance increases because of the smaller distance between measurement point and ground plane, while the inductance decreases due to the smaller effective area of the loop. Electrically, the 10 pins of each module are connected in parallel, leading to an effective capacitance of the printed circuit of $26 \mathrm{pF}$ including the LEMO socket. For the 10 loops, connections both in parallel and in series were examined. Parallel loops provide superior frequency response to at least 100 $\mathrm{MHz}$, entailing an effective $\mathrm{PCB}$ inductancc of the printed cir- cuit of $35.5 \mathrm{nH}$. Serial loops constitute a more suitable load at frequencies below $20 \mathrm{MHz}$, but display a resonance above $50 \mathrm{MHz}$, as can be seen from Fig.3.

\section{SINGLE PIN AND LOOP FIELD PROPERTIES}

The electric and magnetic fields generated by pins or loops may be rigorously calculated in planar geometry $[4,5]$. These results, of course, will provide only a rough estimate for the actual situation in cylindrical geometry.

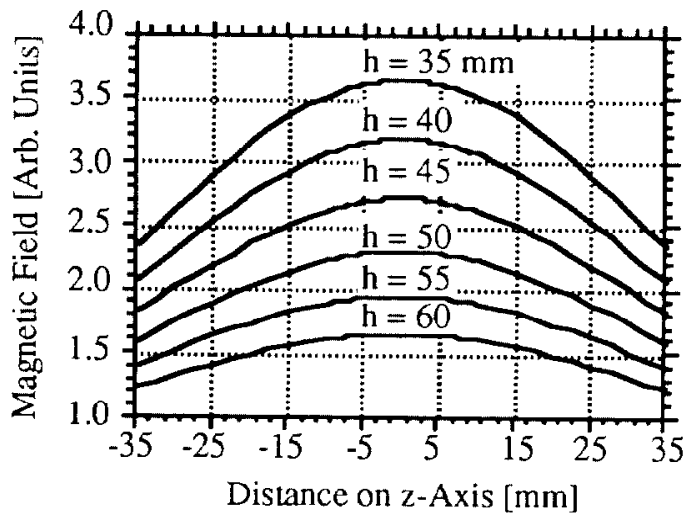

Fig.4: Spatial variation of magnetic field $B_{\phi}$

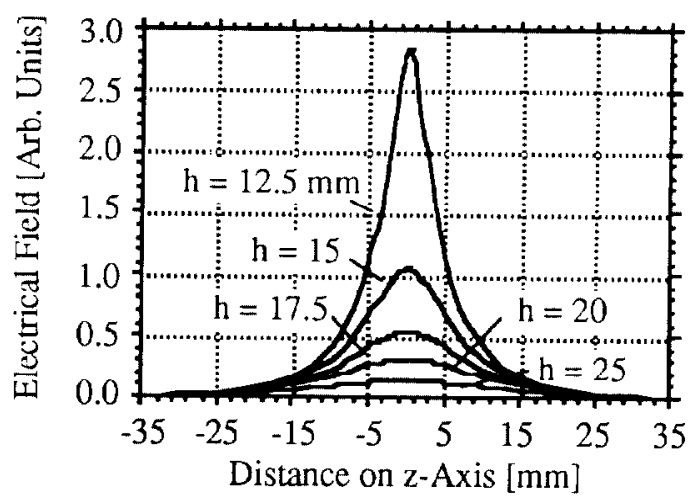

Fig.5: Spatial variation of electric field, $E_{r}$

Figures 4 and 5 show the spatial variation of the respective field components $E_{r}$ and $B_{\phi}$ along the axial direction, for various distances $h$ away from the reference plane. Similar variations can be found along the azimuthal direction. However, the loops or pins around the azimuth are spaced such, that the resulting field ripple does not exceed $20 \%$, leading to ten loops or pins. To derive a shape weighting factor for the field variation along the axis, we integrate the area under the curves, $\int \mathrm{F}(\mathrm{h}) \mathrm{dz} /\left[\mathrm{F}_{\text {peak }}(\mathrm{h}) \times \lambda\right]$, from $-\lambda / 2$ to $\lambda / 2$. For $\lambda_{\min }=$ $35 \mathrm{~mm}$ and $\mathrm{h} \rightarrow 42 \mathrm{~mm}$, the distance to the outer conductor, we get for this weight factor 0.7 (pins), and 0.9 (loops). These factors will be used for the calibration procedure below.

\section{MEASURFMENT SET-UP}

We use the standard technique with a network analyzer (HP $8753 \mathrm{C}$ ) to both generate the signal to be injected into the structure, and to measure and display the transmitted signal, 
see "1" of Fig. 6. Both delays and the field strength ratio have to be adjusted so as to match the desired $\beta$.

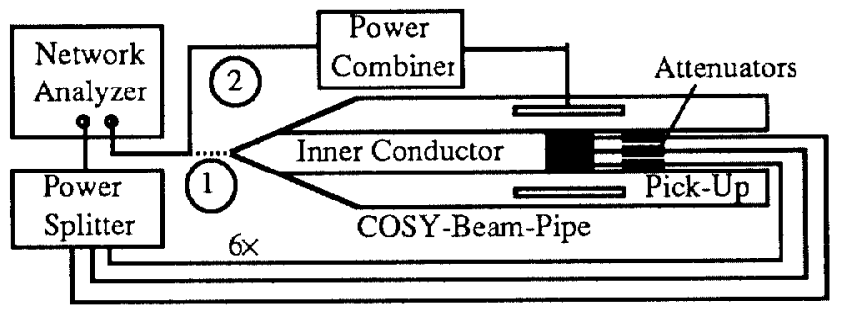

Fig.6: Set-Up

\subsection{Delay lines and phase advance}

To each of the ten electric field modules and ten magnetic field modules there goes one signal cable, plugged into a LEMC socket on the PCB, as indicated in Fig. 1. The delay length, d, of each signal cable (signal propagation speed $c_{c}$ ) to the various modules is adjusted such that the resulting phase velocity of the generated mode corresponds to the desired beam velocity $\beta c_{y}$, i.e. $d=(\lambda / \beta)\left(c_{c} / c_{y}\right)$, where, using solid PE insulated RG174 coax, $\left(c_{c} / c_{v}\right) \sim 0.62$. Thus, for the $(\beta=1)$ measurement with loops and pins, one chooses suitable delays of $\mathrm{d}(\beta=1)=0.62(\lambda / 2)=22.3 \mathrm{~mm}$. An attenuator $(6 \mathrm{~dB})$ at the LEMO socket of each array reduces reflections (Fig. 6).

\subsection{Field calibration and field ratio}

We must adjust, according to Faraday's law of inductance, the field ratio to be $B_{\phi} / E_{r}=\beta / c_{v}=0.33 \times 10^{-8} \times \beta[\mathrm{s} / \mathrm{m}] .[2,4,5]$ First we may determine the properties of the $50-\Omega$ coaxial configuration without any loops or pins installed, i.e. we inpose TEM modes propagating at the speed of light $(\beta=1)$. On both ends, the structure is properly matched into type $\mathrm{N}$ connectors by conical tapers. Both field types are picked up at the inside of the outer (beam) tube via a test pin and a semicircular test loop of the same dimensions as in Sect. 2., protruding radially into the space between inner and outer tube. Feed-through and connection is made by SMA sockets.
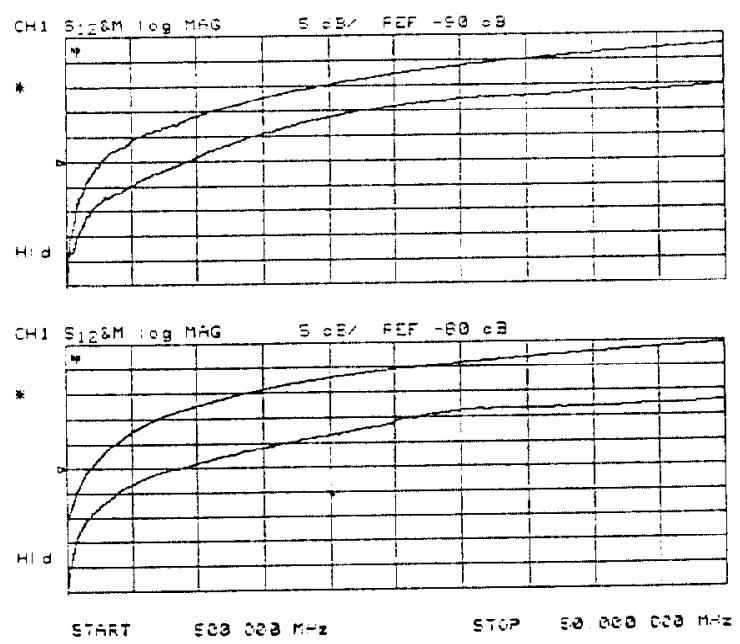

Fig.7: E-field calibration (top); B-field calibration (bottom)
To avoid electrical coupling, i.e, to select exclusively a differential (i.e. magnetic) pick-up from the magnetic test loop, a balanced $180^{\circ}$ splitter (center-tapped matched transformer) was used. First, the electric test loop was aligned with an exciting electric loop on the inner conductor. We used pin-andloop arrays with three modules of each type. The pick up is compared to the result of the coaxial $50 \Omega$ configuration (Fig. 7a). Thereafter, the magnetic test loop was aligned and measured similarly with a magnetic loop (Fig. 7b). Thus, we may generate with the presented pin-and-loop array $(\beta=1)$ TEM modes in an empty coaxial structure, if we attenuate the signals into the pins, relative to the signals into the loops, by $|\mathrm{B}|[\mathrm{dB}]-[\mathrm{E} \mid[\mathrm{dB}]=2.8 \mathrm{~dB}$.

\subsection{Test-measurements with a beam-position monitor}

For arbitrary-beta beam current measurements, a number of current monitors of various geometries are used. For preliminary tests of our low-beta simulation structure ("2" of Fig. 6), we use a standard (spare) COSY beam-position monitor with the common mitre-shaped (diagonally-cut) cylindrical pick-up plate surfaces (diameter as COSY tube). This structure possesses a band pass characteristic to well beyond $100 \mathrm{MHz}$. A (more interesting) strip-line type pick-up with characteristics that can easily be calculated, is contemplated. However, since no spare exists, such a strip-line pick-up would have to be freed specifically for such tests. For the tests presented here, we aligned the axial center of one electric pin module (with 10 azimuthally mounted pins) and the axial center of the BPM.

To reduce noise, we choose a signal of $10 \mathrm{dBm}$ power at the network analyzer generator, and tune the IF bandwidth to $10 \mathrm{~Hz}$, or use a suitable averaging factor. At a frequency of $500 \mathrm{kHz}$ we observe a transmission loss of about $90 \mathrm{~dB}$; at 50 $\mathrm{MHz}$ the transmission loss is $50 \mathrm{~dB}$. Thereafter, the amplitude remains constant to at lcast $100 \mathrm{MHz}$. The drop in magnitude at the lower frequency end is due to the capacitive nature of the BPM, in connection with a low impedance measurement. The electrostatic pick-up plates of our BPM can only register electric signals. To measure the magnetic fields as well, we use the same setup as for calibration, Sect. 5.2.

\section{CONCLUSION}

Wc have devised a technique to realize coaxial modes with any ratio of electric-to-magnetic field and with arbitrary phase velocity, including greater than the speed of light. Field configurations of the Lienard-Wiechert type [4], which are relevant for longitudinal field modes, can be simulated specifically by a suitable choice of the phase velocity and field ratio. With the presented set-up, low-beta beam simulations are possible, both for line charge and longitudinal impedance measurements. As a next step, we will test with this set-up several of the COSY diagnostic structures, as they are available.

\section{REFERENCES}

\section{[1] F. Caspers, Workshop Impedance and Current Limita tions, Grenoble 1988.}

[2] F. Caspers, US-CERN School Hilton Head Is., SC 1990.

[3] F. Caspers, D. Möhl, A. Schwinn, EPAC 1990, Nice.

[4] J.D.Jackson, Classical Electrodynamics, J. Wiley 1975.

[5] Ch. Günther, Diploma Thesis, RWTH Aachen, 1993. 